\title{
Landmark guided internal jugular vein catheterization in infants undergoing congenital heart surgery
}

\section{Serkan Seçiciఠ}

Department of Cardiovascular Surgery, Division of Pediatric Heart Surgery, Bursa Yüksek İhtisas Training and Research Hospital, Bursa, Turkey

\section{ABSTRACT}

Objectives: Central venous catheterization is an important procedure for infants undergoing heart surgery. Ultrasound-guided methods have been shown to decrease failure and complications. The goal of the present study was investigate the effect of anatomical landmark technique during internal jugular vein access in lowweight infants undergoing congenital heart surgery.

Methods: A total 98 infants (median age was 16.5 days, median weight was $3275 \mathrm{~g}$ ) who underwent internal jugular vein catheterization between January 2017 and October 2019 were retrospectively analyzed. All catherizations were performed by a pediatric heart surgeon. Data including demographic characteristics of the infants, success rate, and catheter-related adverse events were recorded.

Results: Success rate was $94.9 \%(n=93)$. There were three arterial puncture $(3.1 \%)$ and hematoma occurred in five patients $(5.1 \%)$. Central venous catheterization of $78(79.6 \%)$ patients were successful at the first attempt.

Conclusions: Our study suggest that landmark technique still seems useful by experienced operators and is safe and effective in infants weighing less than $5 \mathrm{~kg}$ undergoing congenital heart surgery.

Keywords: Central venous catheterization, internal juguler vein, infants

Oen entral venous catheterization has vital importance during congenital heart surgery as well as postoperative care. Two techniques were defined for placement of central venous catheters (CVC): the traditional "landmark" approach or an ultrasound (US)-guided approach. The ultrasound guidance is recommended for locating the vascular structures and guiding the venous puncture, both in adults and in children. The use of US has been shown to increase the rate of success and reduce complications in many studies in which CVCs were placed by non-surgeons [1-5].

However, many surgeons continue to use the anatomical landmark (LM) technique rather than USguidance, because three-dimensional, anatomy-based procedures constitute a significant part of the training process for surgeons, enabling them to be more facile with anatomy-based techniques for insertion of percutaneous CVCs compared with practitioners trained in other specialties [6].

Central venous catheter placement in neonates and small infants is considered a more challenging procedure. In such cases, vein size is small, advancement of the guidewire is often troublesome and puncture area is limited.

In the present study, we aimed to evaluate the clin- 
ical effectiveness and safety of internal jugular vein (IJV) catheterization performed by a pediatric cardiovascular surgeon using LM technique in newborns and infants weighing less than $5 \mathrm{~kg}$.

\section{METHODS}

The study protocol was approved by the Local Ethical Review Committee. All neonates and infants with weight below $5 \mathrm{~kg}$ who had IJV catheterization due to congenital heart disease between January 2017 and October 2019 were retrospectively analyzed.

Data including demographic characteristics of the infants such as age, gender, and body weight, catheterrelated adverse events, surgical procedural details were recorded.

All cannulations were performed by a single pediatric cardiovascular surgeon under general anesthesia, after intubation in the operating room. Patients in this study were consecutive cases of central venous cannulation by the author.

Standard monitoring (electrocardiogram, blood pressure, and pulse oximeter) were applied to all patients. After orotracheal intubation, the patient was positioned with rolled towel under the shoulder with head slightly toward the left side (no more than $40^{\circ}$ ) (Fig. 1). The neck is fixed in more extension than normal position which helps to expose Sedillot's triangle, approximate the IJV to skin and, enables puncture of proximal IJV. For IJV cannulation, there are three approaches consisting of anterior, central, and posterior. In this study, central approach was preferred. The surface anatomy is comprised of the borders of Sedillot's triangle formed by sternal and clavicular heads of the sternocleidomastoid muscle, and the superior border of the medial third of the clavicle inferiorly [7]. The internal jugular vein lies posterior to the Sedillot's triangle. Cutaneous puncture was done at the midpoint of this triangle, directed toward the ipsilateral nipple at an angle $40-45^{\circ}$ with the skin. Once a flash of blood encountered, standard Seldinger technique was followed.

A pass of the needle through the skin was defined as a single puncture attempt. Failure was described as a procedure where the operator could not place the catheter into the IJV, or venous flash could not be provided after 3 attempts. More than 3 attempts were not performed as complication rates are highest with more than 3 cannulation attempts [8].

The number of attempts, success rate, incidence of complications (i.e., arterial puncture, hematoma, pneumothorax, hemothorax, malposition of the catheter) were recorded.

\section{Statistical Analysis}

Statistical analysis was performed using the SPSS for Windows version 22.0 software (SPSS

Inc., Chicago, IL, USA). The KolmogorovSmirnov test was used to test the normal distribution of data. Descriptive data were expressed in mean \pm standard deviation (SD), medians and number and frequency $(\%)$. Correlations between variables were tested by the chi-square analysis and Fisher's exact test. The influence of relevant variables on binary data was investigated by the logistic regression model. A $p$ value of $<0.05$ was considered statistically significant.

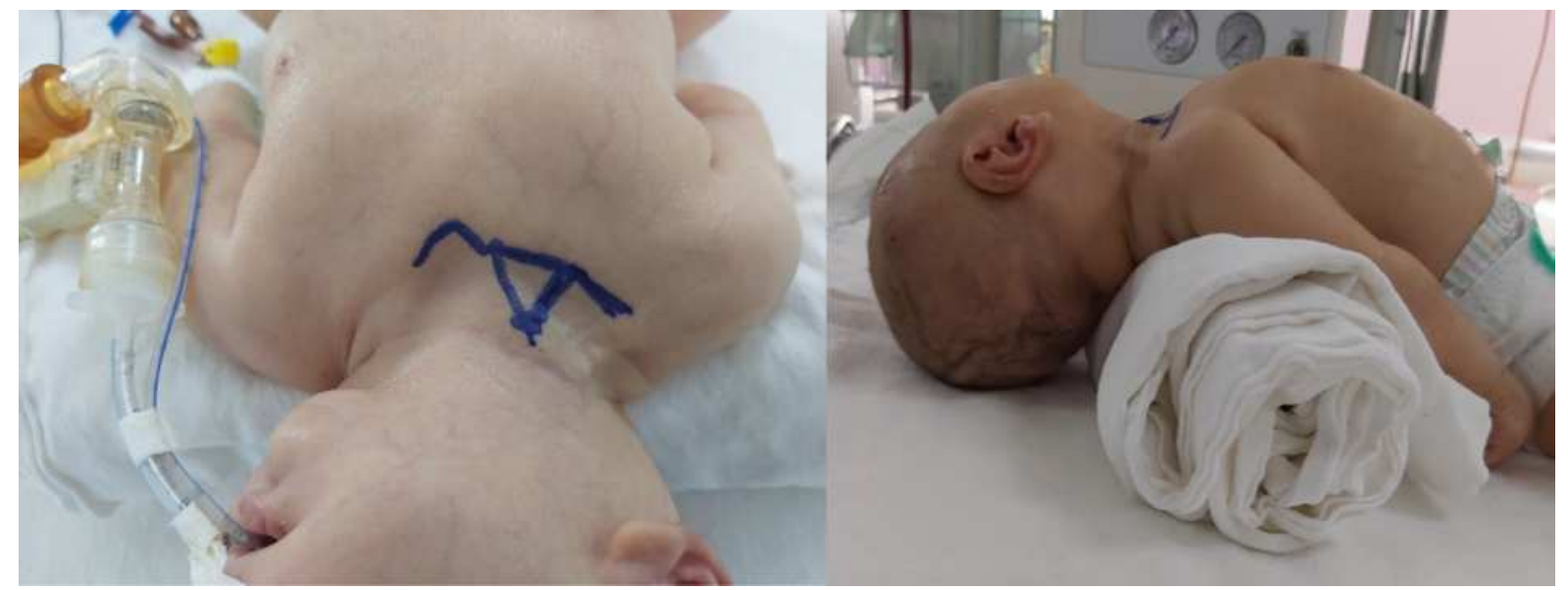

Fig. 1. Position of patient and Sedillot's triangle 


\section{RESULTS}

The patients' weight ranged from 980 to $4999 \mathrm{gr}$ with a median of 3275 gr (25\%-Quartile: 2700 gr; $75 \%$-Quartile: $4000 \mathrm{gr}$ ). The weight was not normally distributed (Kolmogorov-Smirnov test: $p<0.01$ ). 21 patients $(21.4 \%)$ were under 2501 gr. Median age was 16.5 days (2-315). 61 patients $(62.2 \%)$ were in neonatal period, and 37 patients $(37.8 \%)$ were older than 30 days (Table 1).

\section{Table 1. Demographic characteristics of patients} (n= 98)

\begin{tabular}{lll}
\hline Characteristics & $\mathbf{n}$ & $\mathbf{\%}$ \\
\hline Age (days) & & \\
$\leq 30$ & 61 & 62.2 \\
$\quad>30$ & 37 & 37.8 \\
Gender & & \\
Male & 55 & 56.1 \\
Female & 43 & 42.8 \\
Weight (g) & & \\
$\leq 2500$ & 21 & 21.4 \\
\hline $2501-4999$ & 77 & 78.6 \\
\hline
\end{tabular}

Successful cannulation of the IJV was $94.9 \%$ (93 of 98). In five patients IJV catheter placement was failed (Table 2). In these patients, four catheters were placed into left subclavian vein, and one was placed into femoral vein. In total, $78 \mathrm{CVC}(79.6 \%)$ were successful at the first attempt and eleven $(13.3 \%)$ at the second attempt, while seven patients $(7.1 \%)$ required three punctures. Hematoma occurred in five patients (5.1\%). Malposition of the catheter was observed in two patients during operation.

\section{Table 2. Outcomes}

\begin{tabular}{lccc}
\hline & $\begin{array}{c}\mathbf{5 2 5 0 0} \mathbf{g} \\
(\mathbf{n = 2 1 )}\end{array}$ & $\begin{array}{c}\mathbf{2 5 0 1 - 4 9 9 9} \mathbf{g} \\
(\mathbf{n}=\mathbf{7 7})\end{array}$ & Total \\
\hline Failure & 2 & 3 & 5 \\
Arterial Puncture & 2 & 1 & 3 \\
Hematoma & 2 & 3 & 5 \\
Malposition & 1 & 1 & 2 \\
Pneumothorax & 0 & 0 & 0 \\
\hline
\end{tabular}

The patients were divided into two groups according to weight: $\leq 2500 \mathrm{~g}$ and $>2500 \mathrm{~g}$. The median weight of these two groups were $2000 \mathrm{gr}$ (980-2500) and 3500 gr (2600-4999), respectively. The success rates of low weight and normal weight groups were $90.5 \%$ vs 96.1 , respectively ( $p=0.582$ ) (Table 2 ). However, complication rate was significantly higher in the low weight group $(13.3 \%$ vs $\% 7.1 p<0.05)$. There was no significant difference between puncture attempts.

\section{DISCUSSION}

Ultrasound guidance for central venous catheter placement has become increasingly common in the past decade [9]. Today, classical LM and US-guided techniques are being used during CVC placement. However, many surgeons continue to use LM technique rather than US guidance. Studies which cite higher rates of complications with LM technique are largely derived from studies performed by non-surgeons accessing the IJV [10-12].

Exposure during vascular access, vascular bypass, and vascular trauma cases provides surgeons with a valuable view of the vascular anatomy that they access during percutaneous CVC insertions. This perspective is unique to surgeons, as other specialists are accustomed to only seeing these vessels as two-dimensional images on US and CT scans [6].

A survey of pediatric surgeons regarding CVC placement practice patterns revealed that pediatric surgeons who continue to use LM technique did not believe that US was necessary or provided any benefit in regard to lowering complications rates [13]. The educational experience and anatomical knowledge may provide surgeons with a level of comfort.

Gurien et al. [14] pointed that pediatric surgeons were more likely to use LM technique and had decreased complication rates. A meta-analysis by Sigaut et al. [15]presented the use of US during CVC insertions in pediatric patients did not show any reduction in complications or failure rate when used by skilled operators. Also, Grebenik et al. [16] reported US guidance did not help experienced pediatric cardiac anesthetists to achieve better success rates in IJV cannulation in children.

In this cohort of 98 patients, internal jugular veins 
were targeted by experienced pediatric cardiovascular surgeon using LTs. Successful cannulation occurred in $95.1 \%$ with a perioperative complication rate of $8.2 \%$. No pneumothorax or hemothorax was observed.

Our failure rate to insert CVC into IJV using LT was $5.1 \%$ which compares favorably with $10.8 \%$ reported by Arujo et al. [3] using LT and 7.2\% reported by Altun et al. [17] using US. Malbezin et al. [18] reported $1.3 \%$ of complication rate while complication was defined: failure to cannulate any vein, hemothorax, pneumothorax, right atrial perforation, extravenous wire positioning and mean weight was $19 \mathrm{~kg}$. Weight under $3 \mathrm{~kg}$ was defined as predictive failure by Malbezin et al. [18]. However, we found no correlation with the weight and the failure rate. While group weighed under $2.5 \mathrm{~kg}$ had higher complication rate (OR 4.5 [1.2-14.5], $\mathrm{p}<0.05$ ). Altun et al. [17] also found higher complication rate in patients under 2.5 $\mathrm{kg}$.

Bruzoni et al. [8] presented a study comparing LM to US-guided central venous access when performed by pediatric surgeons. In their study success at first attempt was achieved in $65 \%$ of patients in the ultrasound group vs $45 \%$ in the landmark group ( $\mathrm{p}=$ 0.021 ). Success rates were $95 \%$ of ultrasound group vs $74 \%$ of landmark group ( $\mathrm{p}=0.0001)$. In this study $79.6 \%$ of CVC were successful at the first attempt, and overall success rate was $94.5 \%$. Bruzoni et al. detected differences in success rate but not complication rates ( $4.5 \%$ in US vs $4.7 \%$ in LM), and arterial puncture was not defined as complication while it is $4.5 \%$ in US vs $8.3 \%$ in LM. And the patients mean age and weight were 5 years and $27 \mathrm{~kg}$ in US while 8 years and $36 \mathrm{~kg}$ in LM. However, in this study mean weight was $3.3 \mathrm{~kg}$ and, mean age was 51 days which makes CVC insertion technically challenging even in experienced hands.

In a meta-analysis to compare outcomes for surgeon-performed with US CVC insertion versus LM presented that US improves success rate for surgeon performed CVC insertion, but no effect on complication rates. Also, studies in this meta-analysis were not designed on infants and underweight patients.

According to Lamperti et al. [19] most of papers did not declare how the experience of operators were achieved, and history of using with landmark technique makes more difficult the learning curve. Furthermore, for achieving the minimal skills, at least 25 successful supervised ultrasound-guided vein accesses required.

The weight of the patients is also critical factors which affect the success and the complication rate. In a study represented by Froehlich et al. [20], there was a negative correlation between the weight of the patient and the success rate. However, we found no correlation with the weight and the failure rate, while there was a negative correlation between the weight and the complication.

Although the author appreciate the ability to image the internal jugular vein with US, there are some restriction of US guided cannulation. First, lack of space may prevent US use in small infants. Second, US device may not be available in some units. Third, intense training is required. In addition view of arterial pulsation may be difficult in small infants.

One of the most common problem in small infants is inability to pass guide wire. However, in our study, guide wires were easily advanced, as position of the neck enables to puncture of proximal IJV which is wider and closer to right innominate vein and vena cava superior. So J shaped guide-wire can be easily advanced.

\section{Limitations}

The limitations of this study include lack of a control group to compare the usual technique of LT technique and retrospective design with small sample size.

\section{CONCLUSION}

In our study, we found that LT technique still seems useful by experienced operators or when limited to the prelocation of the vein especially in small infants. Success rate of this study is high and, comparable with the US guided studies. In conclusion, our results support the theory that specifically trained skilled operators will have low failure rates when using LT technique. Larger studies needed which document experience level of surgeons as operators and the implementation of US technique.

\section{Authors' Contribution}

Study Conception: SS; Study Design: SS; Supervision: SS; Funding SS; Materials: SS; Data Collection and/or Processing: SS; Statistical Analysis and/or 
Data Interpretation: SS; Literature Review: SS; Manuscript Preparation: SS and Critical Review: SS.

\section{Conflict of interest}

The authors disclosed no conflict of interest during the preparation or publication of this manuscript.

\section{Financing}

The authors disclosed that they did not receive any grant during the conduction or writing of this study.

\section{REFERENCES}

1. Asheim P, Mostad U, Aadahl P. Ultrasound-guided central venous cannulation in infants and children. Acta Anaesthesiol Scand 2002;46:390-2.

2. Ares G, Hunter CJ. Central venous access in children: indications, devices, and risks. Curr Opin Pediatr 2017;29:340-6.

3. Breschan C, Platzer M, Jost R, Stettner H, Feigl G, Likar R. Ultrasound-guided supraclavicular cannulation of the brachiocephalic vein in infants: a retrospective analysis of a case series. Paediatr Anaesth 2012;22:1062-7.

4. Thompson ME. Ultrasound-guided cannulation of the brachiocephalic vein in infants and children is useful and stable. Turk J Anaesthesiol Reanim 2017;45:153-7.

5. Oh C, Lee S, Seo JM, Lee SK. Ultrasound guided percutaneous internal jugular vein access in neonatal intensive care unit patients. J Pediatr Surg 2016;51:570-2.

6. Gurien LA, Blakely ML, Crandall MC, Schlegel C, Rettiganti MR, Saylors ME, et al. Meta-analysis of surgeon-performed central line placement: real-time ultrasound versus landmark technique. J Trauma Acute Care Surg 2018;84:655-63.

7. Bannon MP, Heller SF, Rivera M. Anatomic considerations for central venous cannulation. Risk Manag Healthc Policy 2011;4:27-39.

8. Bruzoni M, Slater BJ, Wall J, St Peter SD, Dutta S. A prospective randomized trial of ultrasound- vs landmark-guided central venous access in the pediatric population. J Am Coll Surg 2013;216:939-43.

9. Oulego-Erroz I, Gonzalez-Cortes R, Garcia-Soler P, BalaguerGargallo M, Frias-Perez M, Mayordomo-Colunga J, et al. Ultrasound-guided or landmark techniques for central venous catheter placement in critically ill children. Intensive Care Med 2018;44:61-72.

10. Denys BG, Uretsky BF, Reddy PS. Ultrasound-assisted cannulation of the internal jugular vein. A prospective comparison to the external landmark-guided technique. Circulation 1993;87:1557-62.

11. Gualtieri E, Deppe SA, Sipperly ME, Thompson DR. Subclavian venous catheterization: greater success rate for less experienced operators using ultrasound guidance. Crit Care Med 1995;23:692-7.

12. Hilty WM, Hudson PA, Levitt MA, Hall JB. Real-time ultrasound-guided femoral vein catheterization during cardiopulmonary resuscitation. Ann Emerg Med 1997;29:331-6; discussion 7.

13. Dassinger MS, Renaud EJ, Goldin A, Huang EY, Russell RT, Streck CJ, et al. Use of real-time ultrasound during central venous catheter placement: results of an APSA survey. J Pediatr Surg 2015;50:1162-7.

14. Gurien LA, Blakely ML, Russell RT, Streck CJ, Vogel AM, Renaud EJ, et al. Real-time ultrasonography for placement of central venous catheters in children: a multi-institutional study. Surgery 2016;160:1605-11.

15. Sigaut S, Skhiri A, Stany I, Golmar J, Nivoche Y, Constant I, et al. Ultrasound guided internal jugular vein access in children and infant: a meta-analysis of published studies. Paediatr Anaesth 2009; 19:1199-206.

16. Grebenik CR, Boyce A, Sinclair ME, Evans RD, Mason DG, Martin B. NICE guidelines for central venous catheterization in children. Is the evidence base sufficient? $\mathrm{Br} \mathrm{J}$ Anaesth 2004;92:827-30.

17. Altun D, Nurac SH, Toprak V, Eti EZ. The success rate and safety of internal jugular vein catheterization under ultrasound guidance in infants undergoing congenital heart surgery. Turk Gogus Kalp Damar Cerrahisi Derg 2019;27:23-8.

18. Malbezin S, Gauss T, Smith I, Bruneau B, Mangalsuren N, Diallo T, et al. A review of 5434 percutaneous pediatric central venous catheters inserted by anesthesiologists. Paediatr Anaesth 2013;23:974-9.

19. Lamperti M, Cortellazzi P, Caldiroli D. Ultrasound-guided cannulation of IJV in pediatric patients: are meta-analyses sufficient? Paediatr Anaesth 2010;20:373-4.

20. Froehlich CD, Rigby MR, Rosenberg ES, Li R, Roerig PL, Easley KA, et al. Ultrasound-guided central venous catheter placement decreases complications and decreases placement attempts compared with the landmark technique in patients in a pediatric intensive care unit. Crit Care Med 2009;37:1090-6. 